\title{
Glucomannan Inhibits Rice Gruel-Induced Increases in Plasma Glucose and Insulin Levels
}

\author{
Akihiro Yoshida $^{a, c}$ Takao Kimura ${ }^{a-c}$ Katsuhiko Tsunekawa ${ }^{a-c}$ Osamu Araki ${ }^{a, b}$ \\ Kazumi Ushiki ${ }^{a}$ b Hirotaka Ishigaki ${ }^{a}$ Yoshifumi Shoho ${ }^{a}$ Itsumi Suda a, b \\ Suguru Hiramoto a, b Masami Murakami ${ }^{a-c}$ \\ ${ }^{a}$ Department of Clinical Laboratory Medicine, Gunma University Graduate School of Medicine, Maebashi, Japan; \\ ${ }^{b}$ Clinical Laboratory Center, Gunma University Hospital, Maebashi, Japan; ' Gunma University Center for Food \\ Science and Wellness, Maebashi, Japan
}

\section{Keywords}

Diabetes · Glucomannan · Glucose · Insulin · Rice gruel

\begin{abstract}
Objective: Given the association between diabetes suppression and inhibition of diet-induced elevation in glucose and insulin, we investigated the effects of adding glucomannan to rice gruel on pre- and postprandial glucose and insulin concentrations. Methods: A total of 25 Japanese subjects without a history of diabetes or gastrointestinal disease (all males; aged 37-60 years; body mass index 20.4-31.6) participated in this study. Subjects received a $75-\mathrm{g}$ oral glucose tolerance test ( $75 \mathrm{gOGTT}$ ) and rice gruel containing $0,0.4$, or $0.8 \%$ of glucomannan. Blood samples were then obtained at preload and at 30,60 , and 120 min after receiving $75 \mathrm{~g}$ of glucose or rice gruel with or without glucomannan. Results: After the $75 \mathrm{gOGTT}, 8$ subjects had normal glucose tolerance (NGT), whereas 17 showed a borderline pattern. Moreover, our data showed that greater amounts of glucomannan promoted lesser 30-min postload plasma glucose and insulin levels, with differences being larger in the borderline group
\end{abstract}

karger@karger.com www.karger.com/anm

Karger $\stackrel{\text { ' }}{5}$

BOPEN ACCESS
(C) 2020 The Author(s)

Published by S. Karger AG, Basel

This article is licensed under the Creative Commons AttributionNonCommercial-NoDerivatives 4.0 International License (CC BYNC-ND) (http://www.karger.com/Services/OpenAccessLicense) Usage and distribution for commercial purposes as well as any distribution of modified material requires written permission. than in the NGT group. Conclusions: Glucomannan dosedependently inhibited the rice gruel-induced increase in 30min postprandial plasma glucose and insulin levels. Furthermore, greater inhibitory effects on glucose and insulin elevation were observed in the borderline group than in the NGT group.

(c) 2020 The Author(s)

Published by S. Karger AG, Basel

\section{Introduction}

Considering the increase in the prevalence of type 2 diabetes mellitus (T2DM) and impaired glucose tolerance (IGT) in every country since 1980, preventing the further growth of such populations has become a major issue [1, 2]. In 2016, the Japanese Ministry of Health, Labour and Welfare estimated that 10 million patients in Japan have diabetes, with another 10 million possibly suffering from the same.

Apart from diabetes, the heightened risk for cardiovascular disease (CVD) has also been found to extend to those with IGT [3]. Both IGT and impaired fasting glu- 
cose, which are considered to be intermediate states in glucose metabolism, are very strong risk markers for the development of diabetes and are also associated with increased CVD risk [4]. Nonetheless, analyses from the DECODE dataset demonstrated that patients with IGT had higher hazard ratios for all-cause mortality than those with impaired fasting glucose. Fasting blood glucose alone is not sufficient to predict mortality related to hyperglycemia. It is important to measure the postprandial glucose concentration after glucose challenge when screening for abnormal glucose [5].

Hyperinsulinemia is also associated with increased allcause and cardiovascular mortality [6]. To prevent glucose intolerance and hyperinsulinemia, diet therapy has been considered as the best method. However, several physicians and patients have described barriers involving compliance with diet therapy [7].

Voglibose belongs to a class of antidiabetic drugs known as $\alpha$-glucosidase inhibitors [8] . $\alpha$-glucosidases are a group of key intestinal enzymes involved in carbohydrate digestion. Subjects treated with voglibose, which competitively inhibits a-glucosidase, showed a lowered risk of progression to T2DM compared with those on placebo $[8,9]$. However, considering that voglibose and other antidiabetic drugs need a prescription, healthy individuals cannot access them before being diagnosed with diabetes even as a preventive measure. Therefore, the development of both low-calorie and high-satiety food products is greatly desired.

Studies have attempted to utilize several types of food products to improve glucose tolerance, including guar gum, oat bran, and wheat farina plus oat gum meals, konjac glucomannan supplement, dietary fiber, porridge made with Scoparia dulcis leaf extract, 5-aminolevulinic acid, and soy protein isolate [10-18]. Glucomannan, a soluble fiber derived from Amorphophallus konjac grown in Japan and a number of Asian countries, has been used as an ingredient in traditional Japanese food [19] and is believed to prolong gastric emptying, increase satiety, and reduce body weight. Glucomannan is also believed to decrease food ingestion, thereby preventing increases in cholesterol and glucose concentrations [20].

In Japan, glucomannan is abundant, easily accessible, and incorporated into various food products. Despite its considerable popularity in Japan, only a few studies have demonstrated the immediate suppression of postprandial glucose and insulin secretion among subjects with normal or IGT. The present study, therefore, investigated the postload glucose and insulin levels among individuals receiving rice gruel with or without glucomannan powder.

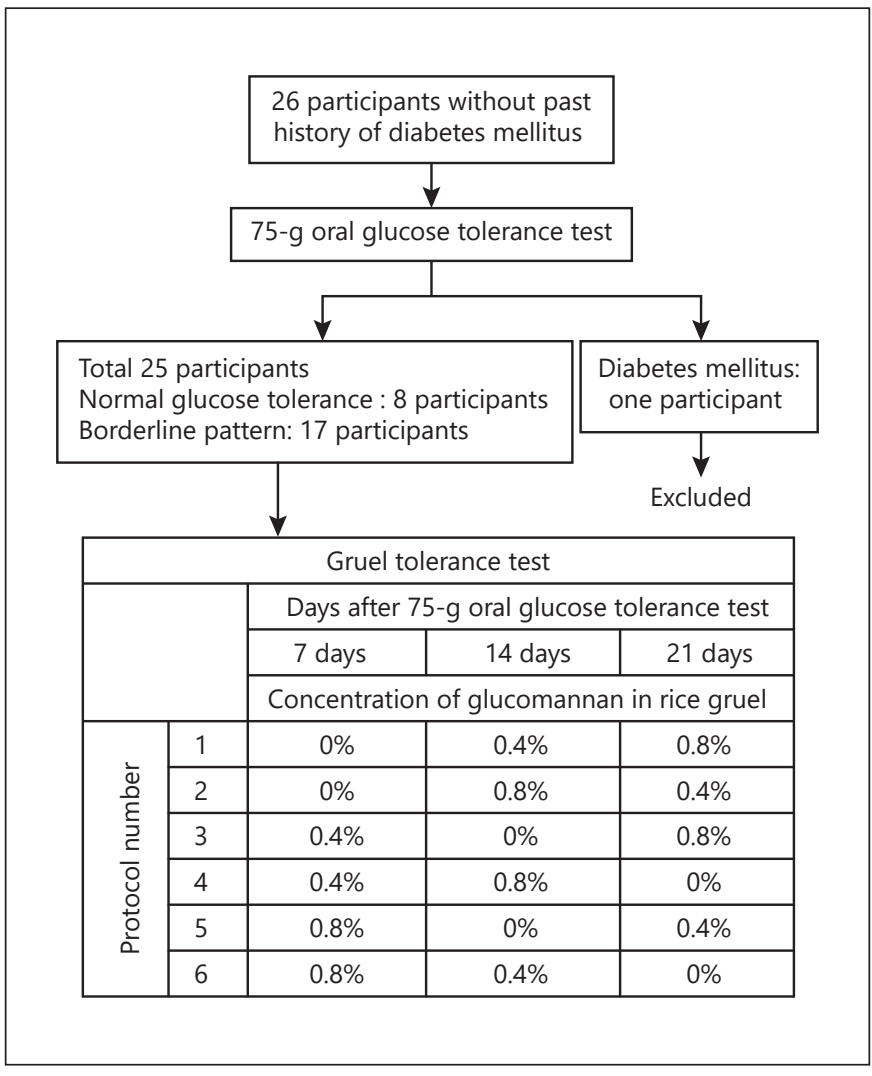

Fig. 1. The design of this study. Twenty-six participants without past history of diabetes mellitus were recruited in this study. All participants received a $75 \mathrm{gOGTT}$. One of the participants had a diagnosis of diabetes mellitus in the $75 \mathrm{gOGTT}$. The participant was excluded from this study because of diabetes mellitus. Thereafter, 25 participants received the gruel tolerance test.

\section{Materials and Methods}

\section{Participants}

All participants provided written informed consent, and the Gunma University Ethical Review Board for Medical Research Involving Human Subjects approved the study protocol (UMIN registration number: UMIN000025950).

A total of 26 Japanese subjects (all males; aged 37-60 years; body mass index 20.4-31.6) participated in this study. Although all the subjects had no history of diabetes or gastrointestinal disease at the beginning of the study, one was diagnosed with diabetes after a 75-g oral glucose tolerance test (75gOGTT) and was therefore excluded.

\section{Test Gruel}

Three types of gruel were used for testing: glucomannan-free rice gruel $(0 \% \mathrm{G})$, rice gruel containing $0.4 \%$ glucomannan powder $(0.4 \% \mathrm{G})$, and rice gruel containing $0.8 \%$ glucomannan powder $(0.8 \% \mathrm{G})$. All the rice gruel preparations were provided by GREEN LEAF Co., Ltd. (Akagihara, Showa, Gunma, Japan). Each type of gruel weighed $250 \mathrm{~g}$, with $0 \% \mathrm{G}, 0.4 \% \mathrm{G}$, and $0.8 \% \mathrm{G}$ containing 75 , 77.5 , and $80 \mathrm{kcal}$, respectively. 


\section{Study Design}

This study used a double-blind, randomized, crossover design, with each subject participating in 3 trials. Figure 1 shows the design of this study. Before the study, all subjects underwent a 75gOGTT after a 12-h overnight fast, with samples collected at $0,30,60$, and 120 min thereafter to establish plasma glucose (PG0, PG30, PG60, and PG120) and immunoreactive insulin (IRI0, IRI30, IRI60, and IRI120) levels. During the preload, serum high-density lipoprotein cholesterol (HDL-C), low-density lipoprotein cholesterol (LDL$\mathrm{C})$, triglycerides, hemoglobin A1c (HbA1c), and glycoalbumin were also measured.

After the 75gOGTT, 25 subjects underwent 3 tests weekly. Each test involved subjects being fed any of the 3 types of gruel followed by blood sampling as with the $75 \mathrm{gOGTT}$. All of 25 participants took 3 concentrations of glucomannan $(0,0.4$, and $0.8 \%)$ in rice gruel within 3 weeks. To perform a double-blind randomized trial, we prepared 6 protocols of gruel tolerance test in this study as described in Figure 1. All of 25 patients were allocated to 1 of 6 protocols at random. Blood sampling was carried out to establish the plasma glucose and immunoreactive insulin levels at preload and at 30,60, and $120 \mathrm{~min}$ after ingesting gruel. Similarly, serum HDLC, LDL-C, triglycerides, HbAlc, and glycoalbumin were also measured during preload.

Laboratory Assays

Serum HDL-C, LDL-C, TG, and GA concentrations were measured using enzymatic methods (LABOSPECT 008; Hitachi, Tokyo), and serum insulin concentrations were measured using chemiluminescence immunoassay (AIA-2000 LA; Tosoh, Tokyo). Plasma glucose concentrations were measured using a hexokinase method (ADAMS Glucose GA-1170; Arkray, Tokyo), and HbA1c levels were measured using high-performance liquid chromatography (ADAMS A1c HA8180; Arkray, Tokyo).

Classification of Glucose Tolerance

Subjects were classified into three groups according to the glucose tolerance category established by the Committee of the Japan Diabetes Society on the Diagnostic Criteria of Diabetes Mellitus [21].

\section{Statistical Analysis}

Statistical analyses were performed using the SPSS version 25 statistical software package. Data were expressed as mean \pm SD.

The effects of time (change from baseline) on blood glucose and plasma insulin concentrations were analyzed using two-way repeated analysis of variance. When a significant effect was detected, the Dunnett and Tukey post hoc tests were conducted to determine the effects of time and treatment, respectively. Statistical significance was set at $p<0.05$.

\section{Results}

The characteristics of all 25 subjects are presented in Table 1. Accordingly, 8 subjects had normal glucose tolerance (NGT), and 17 showed a borderline pattern after the $75 \mathrm{gOGTT}$, suggesting that $68 \%$ of our participants had prediabetes (Table 1). Consistent with the glucose
Table 1. Clinical characteristics and oral glucose tolerance test results

\begin{tabular}{|c|c|c|c|}
\hline & $\begin{array}{l}\text { All } \\
(n=25)\end{array}$ & $\begin{array}{l}\text { NGT } \\
(n=8)\end{array}$ & $\begin{array}{l}\text { Borderline } \\
(n=17)\end{array}$ \\
\hline Age, years & $49.4 \pm 6.6$ & $46.0 \pm 5.3$ & $50.9 \pm 6.8$ \\
\hline $\mathrm{BMI}, \mathrm{kg} / \mathrm{m}^{2}$ & $25.9 \pm 2.9$ & $24.8 \pm 3.9$ & $26.5 \pm 2.4$ \\
\hline LDL-C, mmol/L & $3.18 \pm 0.72$ & $3.47 \pm 0.85$ & $3.05 \pm 0.44$ \\
\hline $\mathrm{HDL}-\mathrm{C}, \mathrm{mmol} / \mathrm{L}$ & $1.22 \pm 0.23$ & $1.27 \pm 0.34$ & $1.19 \pm 0.21$ \\
\hline $\mathrm{TG}, \mathrm{mmol} / \mathrm{L}$ & $1.90 \pm 1.90$ & $1.57 \pm 0.89$ & $2.05 \pm 2.21$ \\
\hline \multicolumn{4}{|l|}{$\mathrm{HbAlc}$} \\
\hline$\%$ & $5.6 \pm 0.3$ & $5.5 \pm 0.3$ & $5.7 \pm 0.3$ \\
\hline $\mathrm{mmol} / \mathrm{mol}$ & $37.2 \pm 0.9$ & $36.1 \pm 0.9$ & $38.2 \pm 0.9$ \\
\hline Glycoalbumin, \% & $13.4 \pm 1.1$ & $13.0 \pm 1.3$ & $13.6 \pm 1.0$ \\
\hline HOMA-IR & $1.73 \pm 0.87$ & $1.42 \pm 0.71$ & $1.87 \pm 0.84$ \\
\hline HOMA- $\beta, \%$ & $59 \pm 27$ & $57 \pm 27$ & $60 \pm 26$ \\
\hline Matsuda index & $5.22 \pm 3.94$ & $7.76 \pm 6.30$ & $4.03 \pm 1.32^{*}$ \\
\hline Insulinogenic index & $0.889 \pm 0.666$ & $1.433 \pm 0.960$ & $0.632 \pm 0.310^{*}$ \\
\hline $\mathrm{PG} 0, \mathrm{mmol} / \mathrm{L}$ & $5.8 \pm 0.4$ & $5.5 \pm 0.3$ & $5.9 \pm 0.3$ \\
\hline PG30, mmol/L & $9.4 \pm 1.4$ & $8.2 \pm 1.2$ & $10.1 \pm 1.2$ \\
\hline PG60, mmol/L & $10.3 \pm 2.4$ & $7.9 \pm 1.6$ & $11.4 \pm 1.9$ \\
\hline PG120, mmol/L & $7.3 \pm 1.8$ & $5.9 \pm 1.3$ & $7.9 \pm 1.6$ \\
\hline IRI0, pmol/L & $48 \pm 23$ & $41 \pm 20$ & $51 \pm 22$ \\
\hline IRI30, pmol/L & $409 \pm 223$ & $502 \pm 357$ & $365 \pm 138$ \\
\hline IRI60, pmol/L & $552 \pm 234$ & $469 \pm 219$ & $592 \pm 216$ \\
\hline IRI120, pmol/L & $404 \pm 221$ & $278 \pm 207$ & $464 \pm 192$ \\
\hline
\end{tabular}

Data are presented as means \pm SD. ${ }^{*} p<0.05$ vs. NGT. NGT, normal glucose tolerance; BMI, body mass index; LDL-C, lowdensity lipoprotein cholesterol; HDL-C, high-density lipoprotein cholesterol; TG, triglycerides; HbA1c, hemoglobin A1c; HOMAIR, homeostasis model assessment of insulin resistance; HOMA- $\beta$, homeostasis model assessment of beta-cell function; PG0, fasting plasma glucose; PG30, 30-min postload plasma glucose; PG60, 60min postload plasma glucose; PG120, 120-min postload plasma glucose; IRI0, fasting plasma insulin; IRI30, 30-min postload plasma insulin; IRI60, 60-min postload plasma insulin; IRI120, 120min postload plasma insulin.

tolerance classification, the borderline pattern group had significantly higher PG0, PG30, PG60, and PG120, as well as IRI120, than the NGT group (Table 1). On the contrary, the borderline pattern group had a significantly lower Matsuda index and insulinogenic index than the NGT group (Table 1). During the $75 \mathrm{gOGTT}$, the plasma glucose and insulin values peaked at $60 \mathrm{~min}$ in all the subjects (Table 1) and in the borderline group (Table 1), whereas the same values peaked at $30 \mathrm{~min}$ in the NGT group (Table 1), indicating delayed glucose-induced insulin secretion among the borderline subjects.

Changes in plasma glucose levels during the rice gruel tolerance test are shown in Figure 2. Among the 3 groups $(0 \% \mathrm{G}, 0.4 \% \mathrm{G}$, and $0.8 \% \mathrm{G})$, no differences in PG0, PG60, 


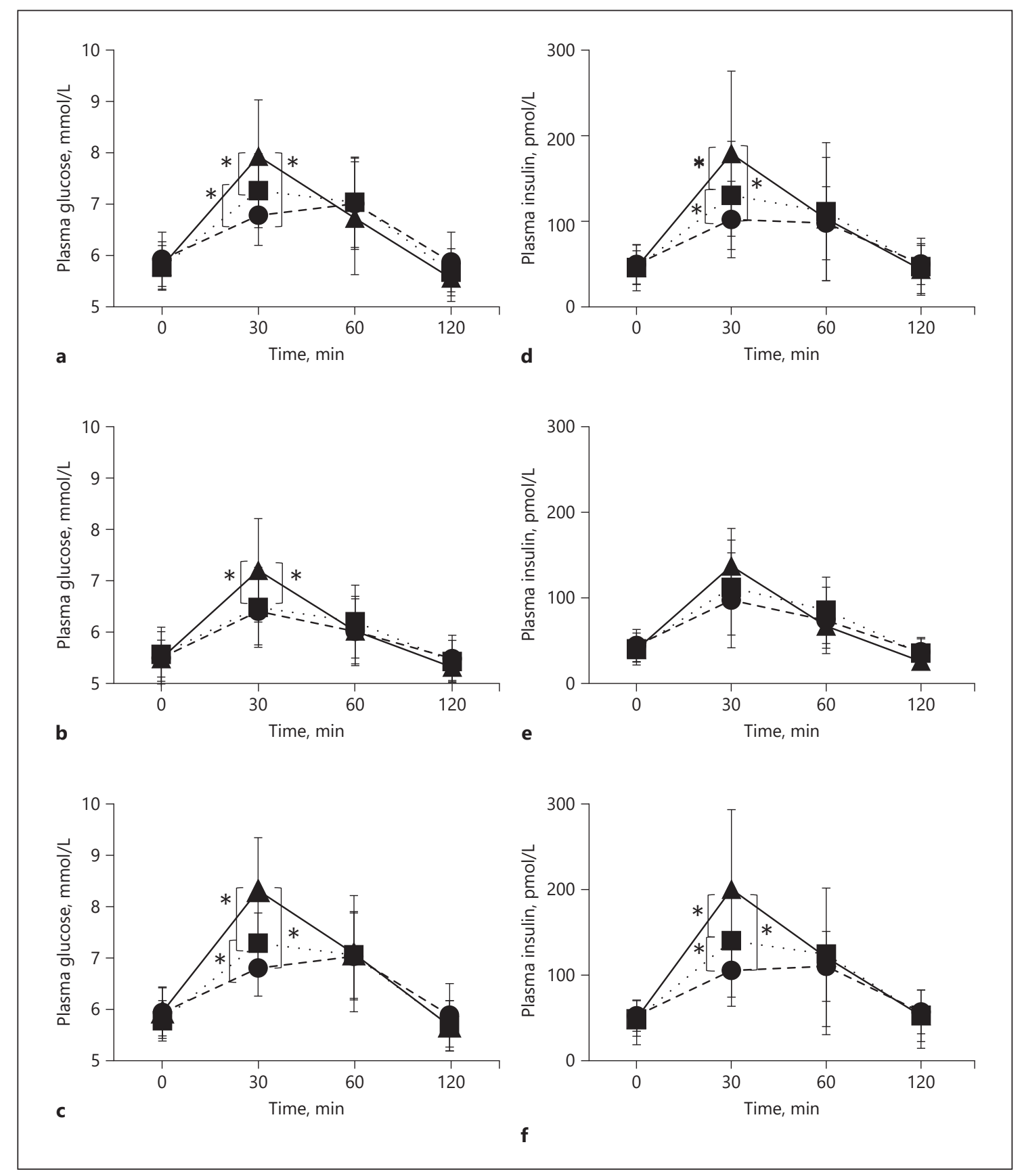

Fig. 2. Sequential changes in plasma glucose and insulin during the gruel tolerance test. Glucomannan concentrations were $0 \%$ (solid triangle), $0.4 \%$ (solid square), and $0.8 \%$ (solid circle) during the $75 \mathrm{gOGTT}$. a Plasma glucose in all subjects, $n=25$. b Plasma glucose in the NGT group, $n=8$. c Plasma glucose in the borderline group, $n=17$. d Plasma insulin in all subjects, $n=25$. e Plasma insulin in the NGT group, $n=8$. $\mathbf{f}$ Plasma insulin in the borderline group, $n=17$. Data are presented as mean \pm SD. ${ }^{*} p<0.05$.

and PG120 were observed (Fig. 2a-c). However, those receiving $0.4 \% \mathrm{G}$ and $0.8 \% \mathrm{G}$ had significantly lower PG30 values ( $6.5 \pm 0.7$ and $6.4 \pm 0.6 \mathrm{mmol} / \mathrm{L}$, respectively) than those receiving $0 \% \mathrm{G}(7.2 \pm 0.9 \mathrm{mmol} / \mathrm{L})$ when consider- ing all subjects, the NGT group, and the borderline group (Fig. 2a-c).

Similar to the glucose values, no differences in IRI0, IRI60, and IRI120 were observed among the 3 groups 


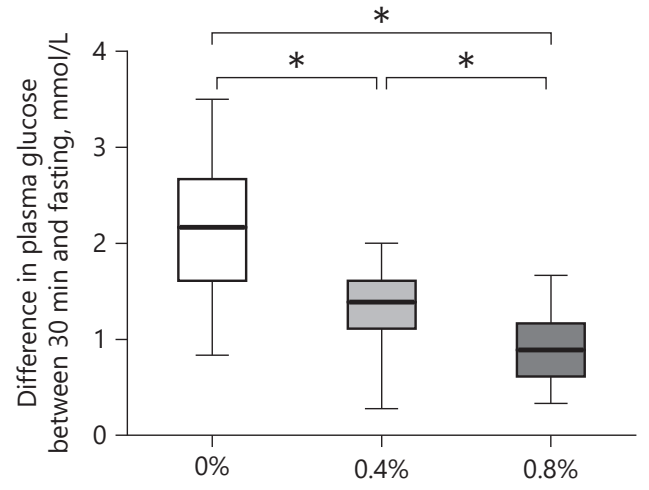

a

Concentrations of glucomannan

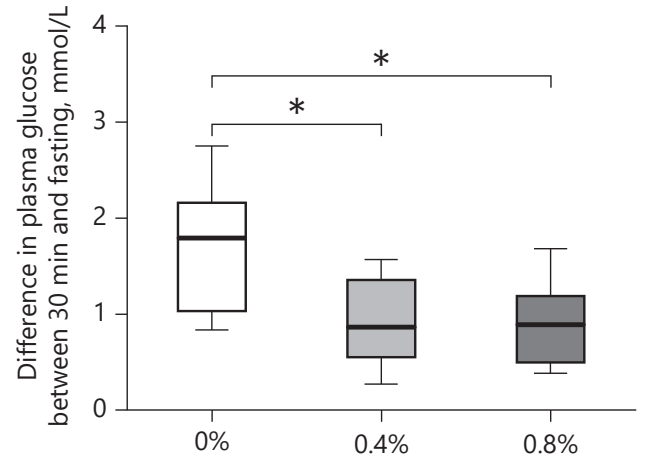

b

Concentrations of glucomannan

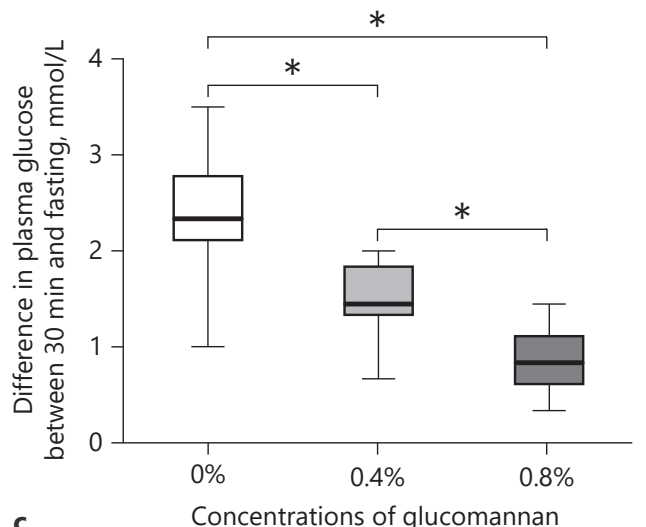

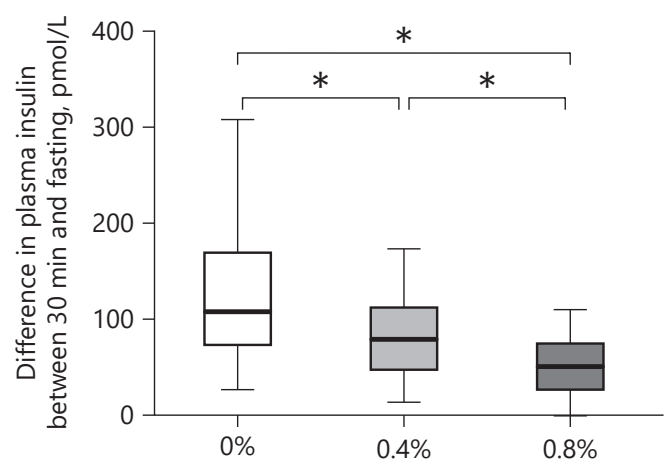

d

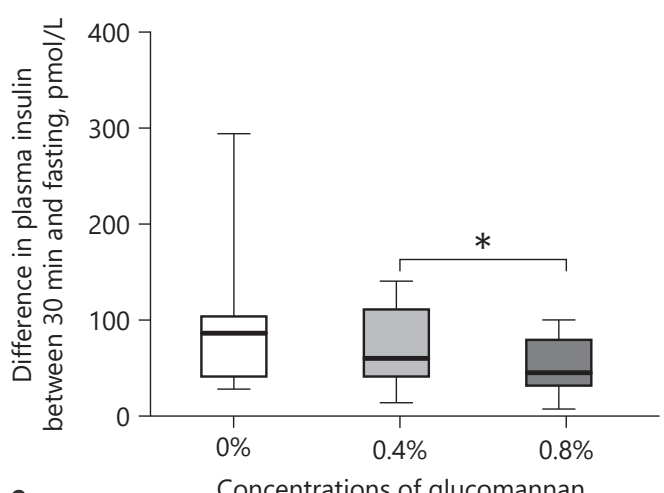

e

Concentrations of glucomannan

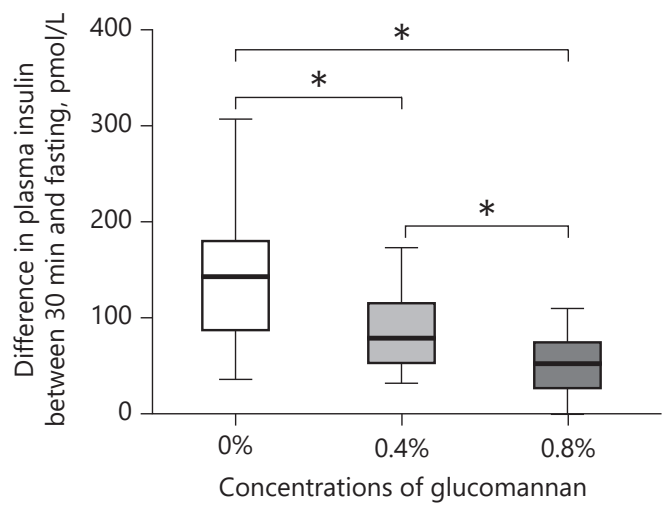

Fig. 3. Box-and-whisker plot of the differences between 30-min postprandial and preload plasma glucose and insulin levels, as well as fasting glucose and insulin levels, during the gruel tolerance test (PG30 - PG0 and IRI30 - IRI0). Glucomannan concentrations were 0\% (open box), $0.4 \%$ (light gray box), and 0.8\% (dark gray box). a Plasma glucose in all subjects, $n=25$. b Plasma glucose in the NGT group, $n=8$. c Plasma glucose in the borderline group, $n=17$. d Plasma insulin in all subjects, $n=25$. e Plasma insulin in the NGT group, $n=8$. $\mathbf{f}$ Plasma insulin in the borderline group, $n=17 . * p<0.05$. 

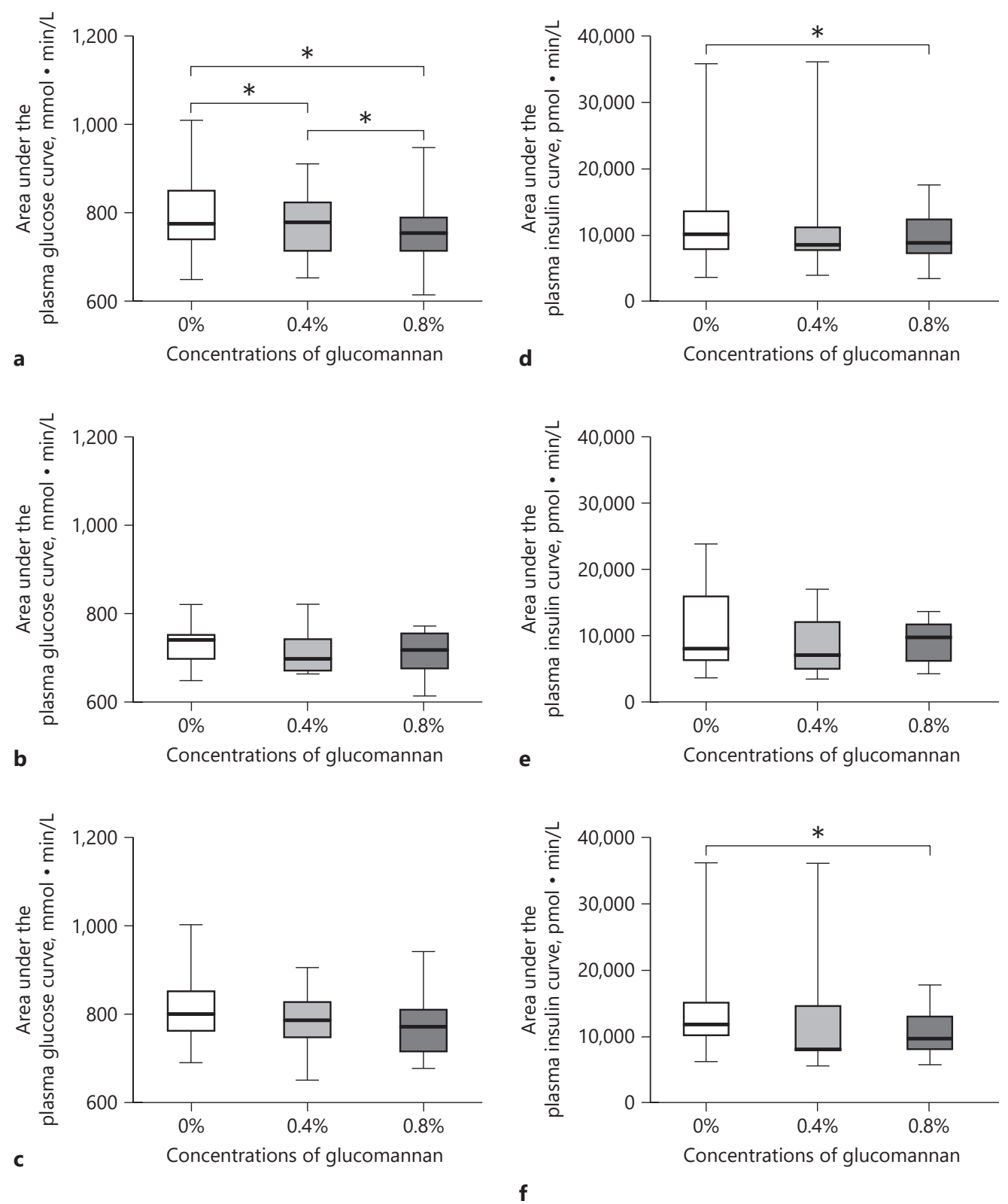

Fig. 4. Box-and-whisker plot of the area under the curve of plasma glucose and insulin during the gruel tolerance test. Glucomannan concentrations were $0 \%$ (open box), $0.4 \%$ (light gray box), and $0.8 \%$ (dark gray box). a Plasma glucose in all subjects, $n=25$. b Plasma glucose in the NGT group, $n=8$. c Plasma glucose in the borderline group, $n=17$. d Plasma insulin in all subjects, $n=25$. e Plasma insulin in the NGT group, $n=8$. $\mathbf{f}$ Plasma insulin in the borderline group, $n=17 .{ }^{*} p<0.05$.

(Fig. 2d-f). Moreover, no differences in IRI30 were observed among those with NGT (Fig. 2e). However, among the borderline group (Fig. 2f) and all subjects (Fig. 2d), those who received $0.4 \% \mathrm{G}$ and $0.8 \% \mathrm{G}$ had significantly lower IRI30 values.
Differences between the 30-min postprandial and preload glucose and insulin levels during the gruel tolerance test (PG30 - PG0 and IRI30 - IRI0) were shown in Figure 3. Significant differences of glucose and insulin levels were observed among $0 \% \mathrm{G}, 0.4 \% \mathrm{G}$, and $0.8 \% \mathrm{G}$ in all sub- 
jects and the borderline group (Fig. 3a, c, d, f). In the NGT group, significant differences in glucose variation were observed among $0 \% \mathrm{G}, 0.4 \% \mathrm{G}$, and $0.8 \% \mathrm{G}$ (Fig. $3 \mathrm{~b}$ ), but not between $0.4 \% \mathrm{G}$ and $0.8 \% \mathrm{G}$. Significant differences in insulin variation were observed only between $0.4 \% \mathrm{G}$ and $0.8 \% \mathrm{G}$ in the NGT group (Fig. 3e).

Significant differences in the areas under the curve of glucose $(\mathrm{AUCg}$ ) were observed among $0 \% \mathrm{G}, 0.4 \% \mathrm{G}$, and $0.8 \% \mathrm{G}$ in all subjects (Fig. $4 \mathrm{a}$ ). Furthermore, areas under the curve of insulin (AUCi) were significantly higher with $0 \% \mathrm{G}$ than with $0.8 \% \mathrm{G}$ (Fig. $4 \mathrm{~d}$ ). However, no significant differences in either glucose or insulin were observed among $0 \% \mathrm{G}, 0.4 \% \mathrm{G}$, and $0.8 \% \mathrm{G}$ in subjects with NGT (Fig. $4 \mathrm{~b}, \mathrm{e}$ ). No significant difference in AUCg was observed among $0 \% \mathrm{G}, 0.4 \% \mathrm{G}$, and $0.8 \% \mathrm{G}$ in the borderline group (Fig. $4 \mathrm{c}$ ). A significant difference in AUCi was only observed between $0 \% \mathrm{G}$ and $0.8 \% \mathrm{G}$ in the borderline group (Fig. $4 \mathrm{f}$ ).

\section{Discussion}

This study is the first to demonstrate that intake of gruel containing glucomannan powder suppresses plasma glucose and insulin elevation. The present study investigated the effects of 2 different glucomannan concentrations on postprandial glucose elevation and insulin secretion among middle-aged Japanese individuals. The addition of glucomannan dose-dependently inhibited the rice gruel intake-induced elevation in plasma glucose and insulin levels at $30 \mathrm{~min}$. The glucomannan-induced inhibitory effects were more apparent among borderline subjects than among those with NGT. Moreover, the inhibitory effects of glucomannan induced a reduction in the AUC of plasma glucose and insulin during the gruel tolerance test. Previous reports have not shown that glucomannan supplements had immediate inhibitory effects against food intake-induced glucose elevation. Together with its supposed ability to prolong gastric emptying time, increase satiety, and reduce body weight, glucomannan is also believed to decrease food ingestion, thereby reducing increases in cholesterol and glucose concentrations [20]. This study may suggest that the gruel form increases the viscosity of gastrointestinal contents, slows gastric emptying, and acts as a barrier to mucosal diffusion to a greater extent than the supplement form. Furthermore, the simultaneous intake of glucomannan and carbohydrates may suppress postprandial plasma glucose elevation without reducing carbohydrate intake. Kashima et al. [18] reported that ingestion of soy protein $30 \mathrm{~min}$ before a $75 \mathrm{gOGTT}$ suppressed glucose elevation, although their results indi- cated a higher insulin response. Accordingly, soy protein stimulated insulin release in $20 \mathrm{~min}$ and sustained the same at least $90 \mathrm{~min}$ after glucose ingestion [18]. In the present study, both glucose elevation and insulin release were simultaneously suppressed by adding glucomannan to gruel. This effect resembles that of $\alpha$-glucosidase inhibitors [9, 22]. The glucomannan-induced significant reduction in the AUC of plasma glucose during the gruel tolerance test was observed among all subjects. On the other hand, the glucomannan-induced significant reduction in the AUC of plasma insulin during the gruel tolerance test was observed between $0 \% \mathrm{G}$ and $0.8 \% \mathrm{G}$, but not $0.4 \% \mathrm{G}$, among all subjects. Thus, the glucomannan-induced inhibitory effects were more apparent for glucose elevation than for that of insulin. These results suggest that supplementation of glucomannan in rice gruel delayed absorption.

Although the mechanism through which glucomannan suppresses plasma glucose still remains unclear, it is presumed to be similar to those of other soluble dietary fibers that increase the viscosity of gastrointestinal contents, slow gastric emptying, and act as a barrier to mucosal diffusion $[20,23]$. Moreover, ingestion of whey protein and glucomannan has been shown to reduce appetite by increasing GLP-1 [24].

Studies have indeed attempted to utilize several types of food products to improve glucose tolerance. One previous study showed that guar gum attenuated blood glucose response in patients with T2DM [10]. Oat bran and wheat farina plus oat gum meals also reduced postprandial plasma glucose excursions and insulin levels in subjects with T2DM [11]. Another study showed that konjac glucomannan supplementation for 28 days improved blood lipid levels and suppressed the elevation of glucose levels in subjects with T2DM [12]. Ingestion of glucomannan for 28 days preprandially attenuated the rise in blood glucose without significantly affecting the insulin levels in subjects with T2DM [13]. In contrast, glucomannan supplements administered to subjects with NGT over 8 weeks were well tolerated but did not promote weight loss, fullness, or improvements in lipid and glucose parameters [14]. Furthermore, dietary fiber induced less plasma glucose elevation than foods not containing dietary fiber among subjects with T2DM [15], whereas porridge made with Scoparia dulcis leaf extract decreased the fasting blood glucose and $\mathrm{HbAlc}$ levels in patients with T2DM [16]. Administration of 5-aminolevulinic acid over 12 weeks reduced the fasting and postprandial glucose levels after a 75gOGTT among subjects with T2DM [17]. Soy protein isolate ingestion at preload improved glycemic control among young healthy subjects [18]. As mentioned previously, glucomannan intake ameliorated glu- 
cose tolerance in subjects with T2DM $[10-13,15,16]$. In contrast, glucomannan capsule intake did not lower plasma glucose and body weight among subjects with NGT [17]. In the present study, glucomannan gruel significantly suppressed glucose and insulin elevation in subjects with NGT and, in particular, a borderline pattern. These differences might be explained by the study protocol. We used a combination of glucomannan and rice gruel, which increases the viscosity of gastrointestinal contents.

Diabetes has been identified as an independent risk factor for CVD mortality [25]. Diabetes and its complications place a considerable burden on health-care financing worldwide [2], with deaths due to CVD also being a severe problem across the globe [25]. Therefore, reducing the number of individuals with IGT, which causes CVD, is imperative. In conclusion, our findings show that adding glucomannan to principal food sources may suppress the increase in the number of individuals with IGT.

\section{Acknowledgments}

All the rice gruel preparations were provided by GREEN LEAF Co., Ltd. (Akagihara, Showa, Gunma, Japan). We thank Mayumi Nishiyama and Tetsuo Machida for their technical assistance and helpful discussion.

\section{Statement of Ethics}

All participants provided informed consent, and the Gunma University Ethical Review Board for Medical Research Involving Human Subjects approved the study protocol (UMIN registration number: UMIN000025950) according to the Helsinki Declaration.

\section{Conflict of Interest Statement}

The authors declare that they have no competing interests to disclose.

\section{Funding Sources}

This work was supported by a Grant-in-Aid for Gunma University and Society Collaboration on Food Science and Wellness to A.Y. and T.K. This work was supported, in part, by Grants-inAid 17H04109 (to M.M.) and 26460641 (to T.K.) for scientific research from the Ministry of Education, Culture, Sports Science, and Technology of Japan.

\section{Author Contributions}

A.Y., T.K., and M.M.: conceptualization. A.Y., T.K., and K.T.: data curation. A.Y., T.K., and K.T.: formal analysis. A.Y., K.T., T.K., and M.M.: funding acquisition. A.Y., T.K., K.T., O.A., K.U., H.I., Y.S., and S.H.: investigation. A.Y., T.K., K.T., O.A., K.U., H.I., Y.S., and S.H.: methodology. M.M.: supervision. A.Y., T.K., K.T., O.A., K.U., H.I., Y.S., and S.H.: validation and visualization. A.Y., T.K., and M.M.: drafting and writing of the original article. T.K. and M.M.: writing - review and editing. All authors read and approved the final manuscript.

\section{Availability of Data and Material}

The datasets generated during and/or analyzed during the current study are available from the corresponding author on reasonable request. All data generated or analyzed during this study are included in this published article.

\section{References}

1 Harris MI. Diabetes in America: epidemiology and scope of the problem. Diabetes Care. 1998 Dec;21 Suppl 3:C11-4.

2 Zhou B, Lu Y, Hajifathalian K, Bentham J, Di Cesare M, Danaei G, et al.; NCD Risk Factor Collaboration (NCD-RisC). Worldwide trends in diabetes since 1980: a pooled analysis of 751 population-based studies with 4.4 million participants. Lancet. 2016 Apr;387(10027):1513-30.

3 Tominaga M, Eguchi H, Manaka H, Igarashi K, Kato T, Sekikawa A. Impaired glucose tolerance is a risk factor for cardiovascular disease, but not impaired fasting glucose. The Funagata Diabetes Study. Diabetes Care. 1999 Jun;22(6):920-4.

4 Abdul-Ghani MA, Tripathy D, DeFronzo RA. Contributions of beta-cell dysfunction and insulin resistance to the pathogenesis of impaired glucose tolerance and impaired fasting glucose. Diabetes Care. 2006 May;29(5):1130-9.
5 DECODE Study Group. Glucose tolerance and mortality: comparison of WHO and American Diabetes Association diagnostic criteria. The DECODE study group. European Diabetes Epidemiology Group. Diabetes Epidemiology: Collaborative Analysis of Diagnostic Criteria in Europe. Lancet. 1999 Aug;354(9179):617-21.

6 Pyörälä M, Miettinen H, Laakso M, Pyörälä K. Plasma insulin and all-cause, cardiovascular, and noncardiovascular mortality: the 22-year follow-up results of the Helsinki Policemen Study. Diabetes Care. 2000 Aug;23(8):1097-102.

7 Suzuki T, Takei R, Inoguchi T, Sonoda N, Sasaki S, Kaise T, et al. Clinical significance of barriers to blood glucose control in type 2 diabetes patients with insufficient glycemic control. Patient Prefer Adherence. 2015 Jun;9:837-45.

8 Kawamori R, Tajima N, Iwamoto Y, Kashiwagi A, Shimamoto K, Kaku K; Voglibose
Ph-3 Study Group. Voglibose for prevention of type 2 diabetes mellitus: a randomised, double-blind trial in Japanese individuals with impaired glucose tolerance. Lancet. 2009 May;373(9675):1607-14.

9 Scheen AJ. Is there a role for alpha-glucosidase inhibitors in the prevention of type 2 diabetes mellitus? Drugs. 2003;63(10):933-51.

10 Uusitupa M, Aro A, Korhonen T, Tuunainen A, Sarlund H, Penttilä I. Blood glucose and serum insulin responses to breakfast including guar gum and cooked or uncooked milk in type 2 (non-insulin-dependent) diabetic patients. Diabetologia. 1984 Jun;26(6):453-5.

11 Braaten JT, Scott FW, Wood PJ, Riedel KD, Wolynetz MS, Brulé $\mathrm{D}$, et al. High beta-glucan oat bran and oat gum reduce postprandial blood glucose and insulin in subjects with and without type 2 diabetes. Diabet Med. 1994 Apr;11(3):312-8. 
12 Chen HL, Sheu WH, Tai TS, Liaw YP, Chen YC. Konjac supplement alleviated hypercholesterolemia and hyperglycemia in type 2 diabetic subjects - a randomized double-blind trial. J Am Coll Nutr. 2003 Feb;22(1):36-42.

13 Chearskul S, Sangurai S, Nitiyanant W, Kriengsinyos W, Kooptiwut S, Harindhanavudhi T. Glycemic and lipid responses to glucomannan in Thais with type 2 diabetes mellitus. J Med Assoc Thai. 2007 Oct;90(10):2150-7.

14 Keithley JK, Swanson B, Mikolaitis SL, DeMeo M, Zeller JM, Fogg L, et al. Safety and efficacy of glucomannan for weight loss in overweight and moderately obese adults. J Obes. 2013;2013:610908.

15 Chan EM, Cheng WM, Tiu SC, Wong LL. Postprandial glucose response to Chinese foods in patients with type 2 diabetes. J Am Diet Assoc. 2004 Dec;104(12):1854-8.

16 Senadheera SP, Ekanayake S, Wanigatunge C. Anti-hyperglycaemic effects of herbal porridge made of Scoparia dulcis leaf extract in diabetics - a randomized crossover clinical trial. BMC Complement Altern Med. 2015 Nov;15(1):410.
17 Higashikawa F, Noda M, Awaya T, Tanaka T, Sugiyama M. 5-aminolevulinic acid, a precursor of heme, reduces both fasting and postprandial glucose levels in mildly hyperglycemic subjects. Nutrition. 2013 Jul-Aug;29(78):1030-6.

18 Kashima H, Uemoto S, Eguchi K, Endo MY, Miura A, Kobayashi T, et al. Effect of soy protein isolate preload on postprandial glycemic control in healthy humans. Nutrition. 2016 Sep;32(9):965-9.

19 Behera SS, Ray RC. Konjac glucomannan, a promising polysaccharide of Amorphophallus konjac K. Koch in health care. Int J Biol Macromol. 2016 Nov;92:942-56.

20 Doi K. Effect of konjac fibre (glucomannan) on glucose and lipids. Eur J Clin Nutr. 1995 Oct;49 Suppl 3:S190-7.

21 Seino Y, Nanjo K, Tajima N, Kadowaki T, Kashiwagi A, Araki E, et al.; Committee of the Japan Diabetes Society on the Diagnostic Criteria of Diabetes Mellitus. Report of the committee on the classification and diagnostic criteria of diabetes mellitus. J Diabetes Investig. 2010 Oct; $1(5): 212-28$.
22 Hirose T, Arakawa M, Kawamori R. a-Glucosidase inhibitors as metabolic modulator on patients with IGT or metabolic syndrome. J Japan Diabetes Soc. 2008;51: 833-6.

23 Jenkins DJ, Wolever TM, Leeds AR, Gassull MA, Haisman P, Dilawari J, et al. Dietary fibres, fibre analogues, and glucose tolerance: importance of viscosity. BMJ. 1978 May; 1(6124):1392-4.

24 Sukkar SG, Vaccaro A, Ravera GB, Borrini C, Gradaschi R, Massa Sacchi-Nemours A, et al. Appetite control and gastrointestinal hormonal behavior (CCK, GLP-1, PYY 1-36) following low doses of a whey protein-rich nutraceutic. Med J Nutrition Metab. 2013;6(3): 259-66.

25 Wei M, Gaskill SP, Haffner SM, Stern MP. Effects of diabetes and level of glycemia on allcause and cardiovascular mortality. The San Antonio Heart Study. Diabetes Care. 1998 Jul; 21(7):1167-72. 